\title{
The Molecular Mechanisms of CD19-Negative Relapse in B-Cell Lymphoma after CAR T-Cell Immunotherapy
}

\author{
Zhihong Wu and Weihong Chen* \\ Department of Hematology, The First Affiliated Hospital of Shenzhen University, University/Shenzhen Second People's Hospital, Shenzhen, China
}

*Corresponding author: Weihong Chen, Department of Hematology, The First Affiliated Hospital of Shenzhen University/Shenzhen Second People's Hospital, Shenzhen 518035, China

To Cite This Article: Zhihong Wu, Weihong Chen. The Molecular Mechanisms of CD19-Negative Relapse in B-Cell Lymphoma after CAR T-Cell Immunotherapy. Am J Biomed Sci \& Res. 2021 - 14(2). AJBSR.MS.ID.001965. DOI: 10.34297/AJBSR.2021.14.001965.

Received: 眥 September 06, 2021; Published: 彗 September 13, 2021

\begin{abstract}
The chimeric antigen receptor T cell (CAR T-cell) immunotherapy is the most antitumor ability in relapse/refractory (R/R) hematological malignancies but it still shows a high relapse rate. A few studies have been found that the molecular mechanisms of CD19-negative relapse after CAR T-cell therapy are the CD19 loss or down-regulation in lymphoma, including lineage switching, CD19 gene mutation, selective shearing, and subcloning of CD19-negative cell. The gene rearrangement, fusion genes and IL-6 may be to influent the therapeutic effect of CAR T-cell immunotherapy. The gene mutations of APX5, IKAROS, EBF1, GNA13, SOCS1, TNFALP3, XP01, FLT3 etc. have been currently found after CAR T-cell therapy relapse. The review reports the molecular mechanisms of CD19-negative relapse in B-cell lymphoma after CAR T-cell immunotherapy.
\end{abstract}

Keywords: CD19-negative relapse, B-cell lymphoma, CAR T-cell immunotherapy, Molecular mechanisms

\section{Introduction}

CAR T-cell immunotherapy is a great advance in the treatment of hematologic malignancies. Some researches find that the complete remission rate about 70\%-90\% for relapsed/refractory B-cell acute lymphocytic leukemia [1]. However, a study has also found that relapse rate of lymphoma is about $30-50 \%$ after CAR T-cell treatment. We have reported that there are two main causes for CD19-positive and CD19-negative relapse after CAR T-cell immunotherapy [2-4]. CD19-positive relapse is mainly due to low efficiency and persistence, senescence of CAR T-cell in vivo. And there are also some confounding factors such as: different co-stimulatory, the manufacture methods, various categories and dosage of CAR T-cell, tumor heterogeneity and so on [2,3]. The main mechanisms of CD19-negative relapse after CAR T-cell therapy may be the presence of the loss or down-regulation of CD19 expression. The effects of other molecular, still unclear genetic mutations, and the indirect effects of other cytokines may influent the expression of CD19.

\section{Directly Related Molecular Mechanisms}

The CD19 loss may be due to lineage switch mutation of CD19 gene or selective shearing and subcloning of CD19-negative cells.

\section{Lineage Switch}

The lineage switch is the interconversion of the B-lymphocyte lineage with the myeloid cell lineage. MLL-B-cell acutelymphoblastic leukemia /B-cell precursor ALL is malleable and reversible in mixed leukemia [5], which may occur myeloid transformation under the selective pressure in CAR T-cell immunotherapy. In recurrent cases, deletion or down-regulation of PAX5, IKAROS and EBF1 was found $[6,7]$. PAX5, located on chromosome 9 , is a member of the coding paired-frame (PAX) family of transcription factors. It encodes 50$\mathrm{kD}$ B-cell specific activator protein (BSAP), which is expressed in pre-B and mature B cells [8]. IKAROS, located at chromosome 7, encodes product is a zinc finger structure-containing transcription factor that plays a critical regulatory role in early lymphocyte 
differentiation $[9,10]$. EBF1, located on chromosome 5, encodes platelet-derived growth factor receptor beta, a receptor tyrosine kinase consisting of 1106 amino acids [11]. PAX5, IKAROS and EBF1 are the main regulators of B cells. PU1 and C/EBP- $\alpha$ 's are involved in myeloid expression. The Loss or downregulation of PAX5, IKAROS and EBF1 leads to failure of CD19 expression and continues expression of myeloid cell, which are causing the lineage switch [6,7].

\section{Mutation or Selective Shearing of the CD19 Gene}

The CD19 gene locates on chromosome 16. It has been shown that the DNA region expressing CD19 has mutations on exons 2-5, of which $4 / 5$ are mutations on exon 3 [12]. Among the mutations, the most notable is on exon 2. SRSF3 splicing factor is also involved in CD19 expression. SRSF3, located on chromosome 6, encodes a protein that is a member of the serotonin/arginine (SR)-rich premRNA splicing factor family [13]. The SRSF3 splicing factor is mainly involved in the retention of exon 2. Hence, the down-regulation of SRSF3 splicing factor also leads to the loss or down-regulation of CD19 $[1,4]$.

\section{CD19-Negative Lymphocyte Subcloning}

The clusters of CD19-negetive lymphoma were found in the patients who were before and after the CAR T-cell treatment. The same clusters of cells were found prior to CAR T-cell treatment, which indicated that patients had CD19 negative B-cells present and cloned before CAR T-cell treatment. In addition, the CD19 was monitored in patients of CD19-negative relapse with retained intron 2 showing non-functional CD19 expression. It may be a mechanism that leads to CD19-negative relapse in lymphoma [1].

\section{Possibly Relevant Molecular Mechanisms}

\section{Mutations in ctDNA}

The patients of relapse lymphoma have been found some mutated genes after CAR T-cell treatment. In one study, continuous monitoring results of circulating tumor DNA (ctDNA) in the patients revealed mutations of GNA13, SOCS1, XP01T and TNFALP3 in ctDNA. GNA13, located on chromosome 17, corresponds to a monitoring region of exon1-4. It encodes the Ga13 protein, which acts to regulate cell morphology, contraction, migration and differentiation and maturation [14]. SOCS1, located on chromosome 17, corresponds to a monitoring region of CDS. It is an important member of the suppressor of cytokine signaling (SOCS) protein family. SOCS1 is involved in a variety of acute and chronic inflammatory responses, innate and acquired immune responses, hormone regulation, and the generation and development of many tumors in the body [15]. XP01, located on chromosome 2, corresponds to a monitoring region of exon15-17 It is an important member of the importin $\beta$ family of nuclear export protein receptors, mainly responsible for the nuclear export of some tumor suppressor proteins and growth regulator proteins [16]. TNFALP3 corresponds to a monitoring region of CDS. There are few relevant studies about TNFALP3. The mutations of GNA13, SOCS1, XP01T and TNFALP3 in ctDNA would exist associated with prognosis after CAR T-cell treatment [17].

\section{Gene Rearrangement and Fusion Genes}

Rearrangement of 11q23 occurs to patients with MLL-r, The most common of which is MLL-AF4. At present, fusion gene of ZNF384 in 12q13, as well as internal repeat crosstalk of the FLT3 gene, are also found in patients of relapse with lineage switch. FLT3, located on chromosome 13, encodes a class III receptor tyrosine kinase that regulates hematopoiesis. Activated receptor kinase phosphorylation activates multiple signaling pathways, including apoptosis, proliferation and differentiation of myeloid hematopoietic cells [18]. The gene rearrangement or fusion genes cause relapse of MLL remains to be investigated after CAR T-cell immunotherapy $[5,19,20]$.

\section{Indirectly Related Molecular Mechanisms}

The IL-6gene, encoded a cytokine that functions as inflammation and B-cell maturation, is located on chromosome 7. In a study, MLL patients with $\mathrm{t}(4,11)$ rearrangements who were treated with CAR T-cells developed cytokine release syndrome (CRS) [21]. The patients that occurred CRS developed myeloid relapse while the other patients that were in remission didn't occur CRS. It has also been shown that IL-6 can drive myeloid differentiation of lymphocytes or cloning of myeloid cells. The results show that IL-6 contributes to myeloid transformation in mixed leukemias [6].

\section{Conclusion}

The current research on CD19-negative relapse is thought to be the loss of CD19 after CAR T-cell immunotherapy. The lineage switching, CD19 gene mutation, selective shearing, and subcloning of CD19- negative are thought to result in the loss of CD19. Moreover, the IL-6, mutated and fused genes have also been discovered. Further studies are needed for its relapse mechanism.

\section{Future Perspectives}

CD19 is the most common target in CAR T-cell immunotherapy. CD22 is a target for salvage therapy after Anti-CD 19 CAR T-cell treatment [22]. The bispecific anti-CD20 and anti-CD19 CAR T-cells are also available to treat lymphoma [23]. The loss of surface antigens may lead to relapse. Therefore, research in the molecular field may be the way forward for CAR T-cell immunotherapy. 


\section{Financial Support and Sponsorship}

1. The relapse mechanism of genetic mutation for the relapsed/ refractory lymphoma after CAR T-cell therapy has been approved by Shenzhen Science and Technology Innovation Committee. Award Number: JCYJ20180228163509339. Grant Recipient: Weihong Chen.

2. CAR T-38 cell therapy for disease progression/relapse multiple myeloma has been approved by Shenzhen municipal Health Commission. Award Number: 201606021. Grant Recipient: Weihong Chen.

\section{References}

1. Shah NN, Fry TJ (2019) Mechanisms of resistance to CAR T cell therapy. Nat Rev Clin Oncol 16(6): 372-385

2. Li X, Chen W (2019) Mechanisms of failure of chimeric antigen receptor T-cell therapy. Curr Opin Hematol 26(6): 427-433.

3. Nie Y, Lu W, Chen D, Tu H, Guo Z, et al. (2020) Mechanisms underlying CD19-positive ALL relapse after anti-CD19 CAR T cell therapy and associated strategies. Biomark Res 8: 18.

4. Orlando EJ, Han X, Tribouley C, Wood PA, Leary RJ, et al. (2018) Genetic mechanisms of target antigen loss in CAR19 therapy of acute lymphoblastic leukemia. Nat Med 24(10): 1504-1506.

5. $\mathrm{Hu} \mathrm{T}$, Murdaugh R, Nakada D (2017) Transcriptional and Microenvironmental Regulation of Lineage Ambiguity in Leukemia Front Oncol 7: 268

6. Gardner R, Wu D, Cherian S, Fang M, Hanafi LA, et al. (2016) Acquisition of a CD19-negative myeloid phenotype allows immune escape of MLLrearranged B-ALL from CD19 CAR-T-cell therapy. Blood 127(20): 24062410 .

7. Jacoby E, Nguyen SM, Fountaine T], Welp K, Gryder B, et al. (2016) CD19 CAR immune pressure induces B-precursor acute lymphoblastic leukaemia lineage switch exposing inherent leukaemic plasticity. Nat Commun 7: 12320

8. Holmes ML, Pridans C, Nutt SL (2008) The regulation of the B-cell gene expression programme by Pax5. Immunol Cell Biol 86(1): 47-53.

9. Bottardi S, Mavoungou L, Milot E (2015) IKAROS: a multifunctional regulator of the polymerase II transcription cycle. Trends Genet 31(9): 500-508.

10. Heizmann B, Kastner P, Chan S (2018) The Ikaros family in lymphocyte development. Curr Opin Immunol 51: 14-23.
11. Barberi T, Cui C, Friedman AD (2020) C/EBPalpha induces Ebf1 gene expression in common lymphoid progenitors. PLoS One 15(12): e0244161.

12. Zhang Z, Chen X, Tian Y, Li F, Zhao X, et al. (2020) Point mutation in CD19 facilitates immune escape of B cell lymphoma from CAR-T cell therapy. J Immunother Cancer 8.

13. Zhou Z, Gong Q, Lin Z, Wang Y, Li M, et al. (2020) Emerging Roles of SRSF3 as a Therapeutic Target for Cancer. Front Oncol 10: 577636.

14. Rabilloud T, Potier D, Pankaew S, Nozais M, Loosveld M, et al. (2021) Single-cell profiling identifies pre-existing CD19-negative subclones in a B-ALL patient with CD19-negative relapse after CAR-T therapy. Nat Commun 12: 865

15. Healy JA, Nugent A, Rempel RE, Moffitt AB, Davis NS, et al. (2016) GNA13 loss in germinal center B cells leads to impaired apoptosis and promotes lymphoma in vivo. Blood 127(22): 2723-2731.

16. Ying J, Qiu X, Lu Y, Zhang M (2019) SOCS1 and its Potential Clinical Role in Tumor. Pathol Oncol Res 25(4): 1295-1301.

17. Azizian NG, Li Y (2020) XPO1-dependent nuclear export as a target for cancer therapy. J Hematol Oncol 13: 61.

18. Zhou L, Zhao H, Shao Y, Chen X, Hong R, et al. (2021) Serial surveillance by circulating tumor DNA profiling after chimeric antigen receptor $\mathrm{T}$ therapy for the guidance of $\mathrm{r} / \mathrm{r}$ diffuse large $\mathrm{B}$ cell lymphoma precise treatment. J Cancer 12(18): 5423-5431.

19. Kennedy VE, Smith CC (2020) FLT3 Mutations in Acute Myeloid Leukemia: Key Concepts and Emerging Controversies. Front Oncol 10: 612880

20. Britten O, Ragusa D, Tosi S, Kamel YM (2019) MLL-Rearranged Acute Leukemia with t $(4 ; 11)$ (q21; q23)-Current Treatment Options. Is There a Role for CAR-T Cell Therapy? Cells 8(11): 1341

21. Oberley MJ, Gaynon PS, Bhojwani D, Pulsipher MA, Gardner RA, et al. (2018) Myeloid lineage switch following chimeric antigen receptor T-cell therapy in a patient with TCF3-ZNF384 fusion-positive B-lymphoblastic leukemia. Pediatr Blood Cancer 65(9): e27265.

22. Zhu H, Deng H, Mu J, Lyu C, Jiang Y, et al. (2021) Anti-CD22 CAR-T Cell Therapy as a Salvage Treatment in B Cell Malignancies Refractory or Relapsed After Anti-CD19 CAR-T therapy. Onco Targets Ther 14: 40234037.

23. Shah NN, Johnson BD, Schneider D, Zhu F, Szabo A, et al. (2020) Bispecific anti-CD20, anti-CD19 CAR T cells for relapsed B cell malignancies: a phase 1 dose escalation and expansion trial. Nat Med 26 (10): 15691575 . 